\title{
O PAPEL DO AGENTE COMUNITÁRIO DE SAÚDE NO APOIO MATRICIAL
}

\author{
THE ROLE OF THE COMMUNITY HEALTH AGENT IN MATRIX SUPPORT SALUD \\ EL PAPEL DEL AGENTE DE SALUD COMUNITARIO EN EL APOYO MATRIZ
}

Andressa da Silva Santos ${ }^{1}$, Fernanda de Moura Soares ${ }^{2}$

\begin{abstract}
RESUMO
Este estudo se propõe a compreender as experiências dos Agentes Comunitários de Saúde com a Saúde Mental e o Apoio Matricial na Atenção Primária à Saúde, e através disso explorar as interfaces entre os agentes comunitários de saúde e o matriciamento. O procedimento de escolha para essas compreensões foi a investigação qualitativa por meio de revisão integrativa, sendo que participaram da revisão produções científicas nacionais. Ao analisar o conteúdo coletado, compreendemos as indispensáveis dimensões do cuidado em saúde mental e os equipamentos e métodos que se atrelam ao agente comunitário de saúde como ferramenta de articulação da rede. Desse modo, o apoio matricial na atenção primária mostrou grande capacidade de qualificar e amparar os profissionais, condicionando ainda mais a corresponsabilização entre equipes e a participação dos agentes comunitários de saúde na estruturação da assistência integrada e de qualidade à população no ambiente da Unidade Básica de Saúde.
\end{abstract}

Descritores: Saúde Mental; Agente Comunitário de Saúde; Atenção Primária à Saúde.

\section{ABSTRACT}

This study aims to understand the experiences of Community Health Agents with Mental Health and Matrix Support in Primary Health Care, and through this to explore the interfaces between community health agents and matrix support. The procedure of choice for these understandings was the qualitative investigation through an integrative review in which national scientific productions participated in the review. By analyzing the collected content, the essential dimensions of mental health care and the equipment and methods that are linked to the community health agent as a tool for articulating the network were understood. Thus, matrix support in primary care showed great capacity to qualify and support professionals, further conditioning the co-responsibility between teams, and the participation of community health agents in the structuring of integrated and quality care to the population in the environment of the Basic Unit of health.

Descriptors: Mental Health; Community Health Agent; Primary Health Care.

\section{RESUMEN}

Este estudio tiene como objetivo comprender las experiencias de los Agentes de Salud Comunitarios con Salud Mental y Apoyo Matricial en Atención Primaria de Salud, y a través de esto explorar las interfaces entre los agentes de salud comunitarios y el apoyo matricial. El procedimiento de elección para estos entendimientos fue la investigación cualitativa a través de una revisión integradora en la que participaron producciones científicas nacionales en la revisión. $\mathrm{Al}$ analizar el contenido recolectado, se entendieron las dimensiones esenciales de la atención en salud mental y los equipos y métodos que se vinculan al agente de salud comunitaria como herramienta de articulación de la red. Desse modo o apoio matricial na atenção primária, mostrou grande capacidade de qualificar e amparar os profissionais, condicionando ainda mais a corresponsabilização entre equipes, e a participação dos agentes comunitários de saúde na estruturação da assistência integrada e de qualidade a população no ambiente da Unidade Básica De salud.

Descriptores: Salud Mental; Agente de Salud Comunitaria; Primeros Auxilios.

\footnotetext{
${ }^{1}$ Centro Universitário Christus. Fortaleza, Ceará, Brasil. (0000-0003-1570-6957)

${ }^{2}$ Centro Universitário Christus. Fortaleza, Ceará, Brasil. (0000-0002-2837-7489)
} 


\section{INTRODUÇÃO}

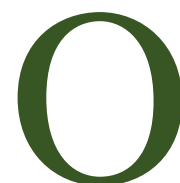

desenvolvimento da Reforma Psiquiátrica no Brasil proporcionou diversas experiências inovadoras e benéficas na prática do cuidado em saúde mental. A evolução de ações de Saúde Pública na esfera da Estratégia Saúde da Família (ESF) possibilitou uma dinamização significativa da atenção, no que se refere À inclusão de assistência às demandas de saúde mental dos usuários no território ${ }^{1}$. Essas experiências na ESF constroem uma perspectiva mais próxima da desconstrução do modelo biomédico privatista, reestruturação do vínculo usuário-profissionalsistema de saúde, além de reduzir o uso indiscriminado de tecnologias duras na rede de atenção à saúde ${ }^{2}$.

A Atenção Primária à Saúde (APS), como coordenadora do cuidado e ordenadora da rede, realiza o redimensionamento dos pacientes para serviços em outros pontos da rede de atenção à saúde, como o Centro de Atenção Psicossocial (CAPS) e hospitais de referência. O encaminhamento sem a contrarreferência pode gerar o distanciamento do usuário da APS, render lacunas na manutenção dos cuidados e progressão do plano terapêutico do mesmo. Diante da situação de afastamento desse usuário do ambiente da atenção primária, o vínculo usuário-profissional fica prejudicado e causa ao profissional a sensação de inexistência de responsabilidade da unidade com o paciente, mesmo essa estando inserida dentro da comunidade a qual o mesmo pertence ${ }^{3}$.

Como possibilidade de articulação entre os equipamentos de saúde, para efetivação da integralidade do cuidado em saúde, o apoio matricial vem fortalecer o cuidado compartilhado ${ }^{4}$. Através do matriciamento, duas ou mais equipes de saúde de diferentes esferas do cuidado podem interagir e compor planos terapêuticos para seus usuários. Essas composições são implantadas, avaliadas e eventualmente alteradas de acordo com a evolução do paciente, as demandas das respectivas equipes e os perfis de usuários que as mesmas contemplam. Desse modo, possibilita-se reunir conhecimentos e buscar informações significativas sobre o indivíduo no seu contexto familiar, pessoal, ocupacional e social. A equipe de matriciadores norteia o cuidado em saúde mental utilizando seus conhecimentos já alicerçados e observando como as intervenções ecoam na vida dos indivíduos. Essa organização de cuidado possibilita a ampliação de saberes articulados e estrutura o entendimento da corresponsabilização dos equipamentos de saúde no cuidado ao paciente em sofrimento mental ${ }^{3}$.

Dentre os profissionais que atuam na ESF, o Agente Comunitário de Saúde (ACS) tem papel fundamental na conexão efetiva da comunidade com o serviço. Ao se posicionar dessa forma no território, o ACS consegue fazer parte do cotidiano dessa população, sendo capaz de transitar entre os equipamentos, dialogar com os profissionais e as pessoas da área. Essa dinâmica de trabalho da classe favorece os vínculos, facilitado o manejo coletivo da assistência ${ }^{5}$.

Reconhecendo o papel do ACS como técnico de referência do território, pode-se vislumbrar que seu exercício no apoio matricial possibilite mais sucesso e melhores resultados para os pacientes em situação de sofrimento mental. Trabalhando nessas perspectivas, Cabral e Albuquerque $^{6}$ evidenciam que a atenção em saúde mental só será trabalhada de maneira efetiva quando as pessoas que fazem parte da rede de apoio fortalecerem a sua articulação entre seus diversos níveis de atenção e estabelecer uma melhor conexão com a própria população.

Ao considerar que se compreende a necessidade de incorporar uma assistência mais ativa do ACS no fortalecimento do apoio matricial à APS, oportunizando a vinculação com a população de cuidados de saúde mental do território, esse estudo parte da seguinte questão norteadora: quais os conhecimentos científicos produzidos na literatura sobre a atuação dos ACS no apoio matricial? 
Diante do exposto, o presente estudo tem por objetivo apresentar as produções científicas nacionais que exploram as interfaces entre os agentes comunitários de saúde e o matriciamento. E, por meio desse, enriquecer a oferta de produções sobre o tema, com intuito de subsidiar as partes da assistência que busquem complementar e amparar a construção do conhecimento acerca do papel do agente comunitário de saúde no apoio matricial e as suas faces como atuante na integração dos serviços, profissionais e clientela dentro da rede de assistência em saúde mental. E desse modo proporcionar o vislumbre de métodos e ferramentas que aprimorem a assistência ao paciente em sofrimento psíquico, e que planeiem maior seguridade aos profissionais que atuam nesse domínio.

\section{METODOLOGIA}

Este é um estudo descrito, do tipo revisão integrativa de literatura, cujo método de pesquisa parte da busca de contribuições científicas, visando sintetizar os resultados obtidos a respeito de uma determinada temática, de forma sistematizada. Desse modo, permite a identificação de lacunas de conhecimentos, direcionamento para futuras pesquisas e a incorporação da aplicabilidade de resultados significativos na prática ${ }^{7}$.

Para tanto, foram executadas as seguintes etapas: 1 - Elaboração da questão de pesquisa; 2 Estabelecimento de critérios de inclusão e exclusão de estudos e busca na literatura; 3- Coleta de dados; 4 - Análise dos dados dos estudos selecionados; 5 Interpretação dos resultados; e 6 - Apresentação de revisão síntese ${ }^{7}$.

Considerando a opção por analisar estudos brasileiros, a composição da amostra foi realizada em fevereiro no ano de 2021, por meio de busca na base de dados da Biblioteca Virtual de Saúde (BVS), englobando a Literatura Latino-Americana e do Caribe em Ciências da Saúde (LILACS) e o Banco de Dados em Enfermagem (BDENF). As buscas foram direcionadas por descritores controlados combinados com operador booleano.
Dessa forma, a estratégia de busca ocorreu com a seguinte chave na busca avançada: "agente comunitário de saúde AND apoio matricial”.

Com relação aos critérios de inclusão para seleção das referências, foram estabelecidas: ser artigo original cujo texto estivesse disponível na integra on-line, que apresentasse conteúdo sobre as experiências de agentes comunitários de saúde no apoio matricial, publicados no idioma português. Quanto aos critérios de exclusão, foram descartadas teses de doutorado, estudos de revisão, artigos repetidos e que não incluíssem o objeto de pesquisa.

Figura 1 - Fluxograma 1 - Processo de seleção de artigos para compor revisão, 2021.

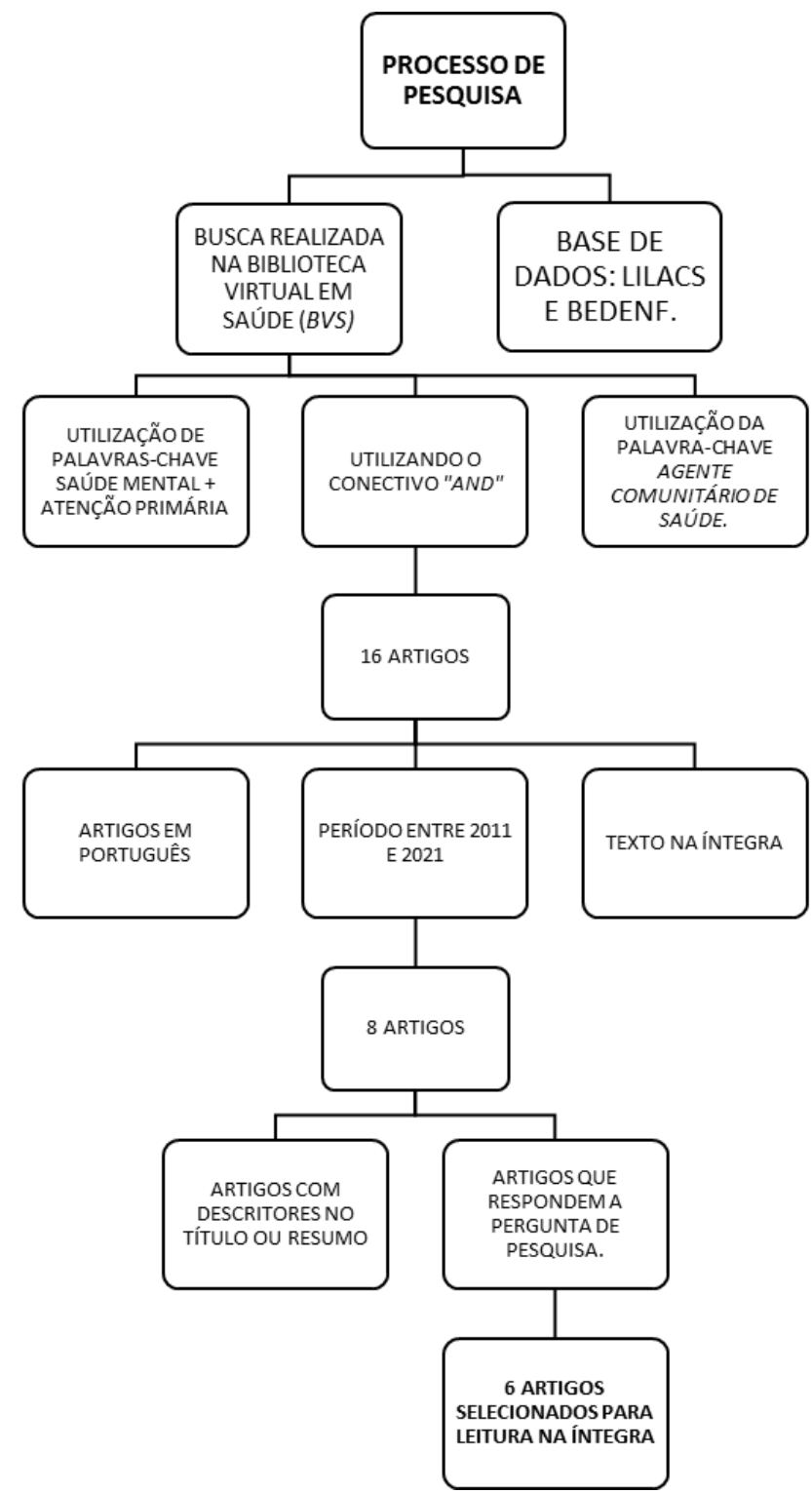

Fonte - Dados coletados pelas pesquisadoras, Fortaleza, Ceará, Brasil, 2021. 
É relevante considerar que devido à escassez de artigos com amostra exclusiva de agentes comunitários de saúde, nesta revisão, considerou-se aqueles estudos mistos compreendendo os ACS e também outros profissionais de saúde.

Inicialmente, foram encontradas 16 publicações utilizando a chave de busca; em seguida, conforme os critérios inclusivos, foram delimitados nove manuscritos. Realizou-se leitura criteriosa dos títulos e resumos. Desses, três foram subtraídos, um por ser uma tese de doutorado e os outros por não contemplarem os objetivos definidos. Por fim, foi realizada nova triagem com a leitura dos textos na integra, sendo seis estudos selecionados para compor a amostra. Essas etapas estão discriminadas no fluxograma.

A partir da leitura criteriosa dos títulos, resumos e palavras-chave, verificou-se a adequação dos estudos aos critérios de inclusão e exclusão e aos objetivos da pesquisa, sendo, a partir disso, realizada a leitura na íntegra das respectivas produções.

\section{RESULTADOS}

Os dados obtidos nos estudos selecionados são apresentados no Quadro 1, sintetizando a identificação do artigo, título, autoria e periódico. No Quadro 2 são apresentados os resultados dos estudos selecionados.

Quadro 1 - Publicações analisadas, segundo título, autoria, periódico e ano. Fortaleza (CE) 2021.

\begin{tabular}{|c|c|c|c|}
\hline ESTUDO & TÍTULO & AUTORIA & PERIÓDICO \\
\hline & APOIO & & \\
& MATRICIAL EM & & \\
& SAÚDE & & \\
& MENTAL NA & AMARAL & \\
& ATENÇÃO & CEM, & \\
$\mathbf{E} 1$ & BÁSICA: & TORRENTE & INTERFACE \\
& EFEITOS NA & MON, & 2018 \\
& COMPREENSÃO & TORRENTE M, & \\
& E MANEJO POR & MOREIRA CP & \\
& PARTE DE & & \\
& AGENTES & & \\
& COMUNITÁRIOS & & \\
& DE SAÚDE. & & \\
\hline
\end{tabular}

\begin{tabular}{|c|c|c|c|}
\hline E2 & $\begin{array}{l}\text { ENTRE OS NÓS } \\
\text { DA SAÚDE } \\
\text { MENTAL : AS } \\
\text { PRÁTICAS DE } \\
\text { AGENTES } \\
\text { COMUNITÁRIOS } \\
\text { DE SAÚDE. }\end{array}$ & $\begin{array}{c}\text { SILVA MAC, } \\
\text { AGUIAR MGG, } \\
\text { MOREIRA TDS } \\
9 .\end{array}$ & $\begin{array}{l}\text { REVISTA BAIANA } \\
\text { DE SAÚDE PÚBLICA } \\
2016\end{array}$ \\
\hline E3 & $\begin{array}{c}\text { SAÚDE } \\
\text { MENTAL NA } \\
\text { ATENÇÃO } \\
\text { BÁSICA: } \\
\text { SENTIDOS } \\
\text { ATRIBUÍDOS } \\
\text { PELOS } \\
\text { AGENTES } \\
\text { COMUNITÁRIOS } \\
\text { DE SAUUDE. }\end{array}$ & $\begin{array}{l}\text { MOURA RFS, } \\
\text { SILVA CRC }{ }^{10} \text {. }\end{array}$ & $\begin{array}{l}\text { PSICOL CIÊNC PROF } \\
2015\end{array}$ \\
\hline E4 & $\begin{array}{c}\text { O CUIDADO EM } \\
\text { SAUUDE } \\
\text { MENTAL PELOS } \\
\text { AGENTES } \\
\text { COMUNITÁRIOS } \\
\text { DE SAÚDE: O } \\
\text { QUE } \\
\text { APRENDEM EM } \\
\text { SEU } \\
\text { COTIDIANO DE } \\
\text { TRABALHO. }\end{array}$ & $\begin{array}{l}\text { SANTOS GA, } \\
\text { NUNES MO }{ }^{11} \text {. }\end{array}$ & $\begin{array}{l}\text { PHYSIS: REVISTA } \\
\text { DE SAÚDE } \\
2014\end{array}$ \\
\hline E5 & $\begin{array}{l}\text { ESTRATÉGIA } \\
\text { DE APOIO EM } \\
\text { SAÚDE } \\
\text { MENTAL AOS } \\
\text { AGENTES } \\
\text { COMUNITÁRIOS } \\
\text { DE SAUUDE DE } \\
\text { SALVADOR BA. }\end{array}$ & $\begin{array}{l}\text { SILVA CB, } \\
\text { SANTOS JE, } \\
\text { SOUZA RC }{ }^{12} \text {. }\end{array}$ & $\begin{array}{l}\text { SAÚDE SOC } \\
2012\end{array}$ \\
\hline E6 & $\begin{array}{c}\text { SAÚDE } \\
\text { MENTAL NA } \\
\text { ATENÇÃO } \\
\text { PRIMÁRIA À } \\
\text { SAÚDE: } \\
\text { ESTUDO } \\
\text { AVALIATIVO } \\
\text { EM UMA } \\
\text { GRANDE } \\
\text { CIDADE } \\
\text { BRASILEIRA. }\end{array}$ & $\begin{array}{c}\text { CAMPOS RO, } \\
\text { GAMA CA, } \\
\text { FERRER AL, } \\
\text { SANTOS DVD, } \\
\text { STEFANELLO } \\
\text { S, TRAPÉ TL, } \\
\text { PORT K }{ }^{13} .\end{array}$ & $\begin{array}{l}\text { CIÊNCIA \& SAÚDE } \\
\text { COLETIVA } \\
\text { [ONLINE] } 2011\end{array}$ \\
\hline
\end{tabular}

Fonte - Dados coletados pelas pesquisadoras, Fortaleza, Ceará, Brasil, 2021.

Quadro 2 - Apresentação dos resultados dos estudos selecionados. Fortaleza (CE), 2021.

\section{ESTUDO} RESULTADOS

E1 O ESTUDO APRESENTOU COMO RESULTADO DIVERSAS TRANSFORMAÇÕES PERCEBIDAS APÓS A UTILIZAÇÃO DA ESTRATÉGIA DE MATRICIAMENTO NA ATENÇÃO PRIMÁRIA A SAÚDE, NO CONTATO DO AGENTE COMUNITÁRIO DE SAÚDE COM A POPULAÇÃO QUE NECESSITA DE ATENÇÃO EM SAÚDE MENTAL NO

TERRITÓRIO. ENTRE AS TRANSFORMAÇÕES OBSERVADAS, OBSERVOU-SE A DIMINUIÇÃO DO TEMOR NA ABORDAGEM AO PÚBLICO, GANHOS NO VÍNCULO PROFISSIONAL-PACIENTE, ALÉM DO ESTREITAMENTO DA COMUNICAÇÃO ENTRE APS E CAPS.

E2 $\quad$ O ESTUDO EXPÕE AS NECESSIDADES E DEMANDAS DOS USUÁRIOS DA ATENÇÃO EM SAÚDE MENTAL DENTRO DA APS, PONDO EM PAUTA SITUAÇÕES DO COTIDIANO DE ATENDIMENTO E DIFICULDADES NO AMPARO A ESSE PÚBLICO, QUE PODE SOFRER COM A BAIXA 


\begin{tabular}{|c|c|}
\hline & $\begin{array}{c}\text { COMPREENSÃO DE PROFISSIONAIS QUE NÃO } \\
\text { ENTENDEM A IMPORTÂNCIA DA ABORDAGEM E DO } \\
\text { CUIDADO SINGULAR, NECESSITANDO DE REGULAR } \\
\text { CAPACITAÇÃO PARA UMA ESCUTA MAIS QUALIFICADA. } \\
\text { O ACS COM UM OLHAR TREINADO CONSEGUE } \\
\text { VISUALIZAR E REPASSAR PARA OS DEMAIS } \\
\text { PROFISSIONAIS DA UNIDADE NECESSIDADES QUE SÓ } \\
\text { SÃO DETECTADAS PELA SUA PROXIMIDADE COM O } \\
\text { TERRITÓRIO. }\end{array}$ \\
\hline E3 & $\begin{array}{l}\text { O ESTUDO APRESENTOU O SENTIMENTO DE TEMOR E } \\
\text { OUTRAS DIFICULDADES DO AGENTE COMUNITÁRIO DE } \\
\text { SAÚDE NO CUIDADO À PESSOA EM SOFRIMENTO } \\
\text { MENTAL, REVELANDO UMA ASSISTÊNCIA AINDA } \\
\text { MUITO PAUTADA NA DOENÇA E NÃO NO USUÁRIO DO } \\
\text { SERVIÇO COMO INDIVÍDUO COM NECESSIDADES } \\
\text { GLOBAIS. DESSE MODO, AS ESCUTAS QUALIFICADAS } \\
\text { DESEMPENHADAS POR RESIDENTES JUNTO ÀS VISITAS } \\
\text { E VIGILÂNCIA CONSTANTE DO ACS POSSIBILITOU UMA } \\
\text { MELHORA SIGNIFICATIVA NA SAÚDE MENTAL DE } \\
\text { ALGUNS USUÁRIOS DA UNIDADE, MOSTRANDO QUE AS } \\
\text { MUDANÇAS NA ABORDAGEM DO INDIVÍDUO SÃO } \\
\text { VALOROSAS PARA A SUA EVOLUÇÃO POSITIVA. }\end{array}$ \\
\hline E4 & $\begin{array}{l}\text { O ESTUDO APRESENTOU OS PRINCIPAIS TÓPICOS } \\
\text { ESTABELECIDOS PELOS ACS, COMO IMPORTANTES NO } \\
\text { DESENROLAR DO CUIDADO A PACIENTES DE SAÚDE } \\
\text { MENTAL NA ATENÇÃO PRIMÁRIA À SAÚDE. DENTRE AS } \\
\text { PRINCIPAIS CARACTERÍSTICAS ELENCADAS PELO } \\
\text { PROFISSIONAL ACS COMO NECESSÁRIAS NESSE } \\
\text { CUIDADO ESTÁ A ESCUTA E ORIENTAÇÃO DA FAMÍLIA, } \\
\text { ESTAR INSERIDO DENTRO DO TERRITÓRIO PARA } \\
\text { CONHECER O CONTEXTO DE VIDA DO INDIVÍDUO E } \\
\text { REALIZAR A ESCUTA DAS QUEIXAS DESSE. }\end{array}$ \\
\hline E5 & $\begin{array}{c}\text { O ESTUDO ABORDA A PARCERIA ENTRE A EQUIPE DE } \\
\text { MATRICIADORES E O ACS NUMA PERSPECTIVA } \\
\text { POSITIVA, QUE PODE TRAZER MUITAS VANTAGENS AO } \\
\text { SERVIÇO E POPULAÇÃO. COM ISSO, FOI } \\
\text { PROPORCIONADA UMA MELHOR COMPREENSÃO DESSE } \\
\text { PROFISSIONAL SOBRE OS PLANOS TERAPÊUTICOS } \\
\text { UTILIZADOS PARA IMPLEMENTAR O CUIDADO AO } \\
\text { PACIENTE EM SOFRIMENTO MENTAL. ALÉM DISSO, A } \\
\text { INFLUÊNCIA DO TRABALHO NA VIDA DO AGENTE } \\
\text { COMUNITÁRIO DE SAÚDE, SENDO ELE O PRINCIPAL } \\
\text { OUVINTE DAS INSATISFAÇÕES DA COMUNIDADE, PODE } \\
\text { GERAR GRANDES TENSIONAMENTOS E AFLIÇÕES NO } \\
\text { MESMO. A PARTIR DOS CONHECIMENTOS REPASSADOS } \\
\text { NO APOIO MATRICIAL, O ACS DE DEIXOU DE AGIR DE } \\
\text { MANEIRA INTUITIVA NOS SEUS ATENDIMENTOS E } \\
\text { PASSOU A SER UM INDIVÍDUO PARTICIPANTE DO } \\
\text { PROCESSO DE CONSTRUÇÃO DA TERAPÊUTICA A } \\
\text { PARTIR DOS SEUS CONHECIMENTOS SOBRE O } \\
\text { CONTEXTO DE VIDA DO INDIVIIDUO EM SOFRIMENTO } \\
\text { PSÍQUICO. }\end{array}$ \\
\hline E6 & $\begin{array}{c}\text { ESSA PRODUÇÃO ABORDOU A COMUNICAÇÃO ENTRE } \\
\text { AS REDES DE ATENÇÃO EM SAÚDE MENTAL, EXPONDO } \\
\text { A DIFICULDADE NO DESENROLAR DA } \\
\text { CORRESPONSABILIZAÇÃO, TENDO EM VISTA QUE ESSE } \\
\text { ARTIFÍCIO DEPENDE DIRETAMENTE DE UMA BOA } \\
\text { COMUNICAÇÃO. OUTRAS PRÁTICAS, COMO A }\end{array}$ \\
\hline
\end{tabular}

\begin{tabular}{|c|c|}
\hline REALIZAÇÃO DE PRESCRIÇÕES REPETIDAS SEM UMA \\
PRÉVIA AVALIAÇÃO DA EVOLUÇÃO DO PACIENTE E \\
SEM CONSIDERAR SUA ADAPTAÇÃO, PODEM TRAZER \\
GRANDES PREJUÍZOS NO QUE SE REFERE AO CONFORTO \\
E QUALIDADE DE VIDA DESSE USUÁRIO, SUBMETENDO \\
O PACIENTE AO MÉTODO DE QUEIXA-CONDUTA, SEM \\
LEVAR EM CONSIDERAÇÃO OUTROS ASPECTOS \\
SIGNIFICATIVOS DO COTIDIANO DO MESMO, TRAZENDO \\
INSATISFAÇÃO NO CUMPRIMENTO DO PLANO \\
TERAPÊUTICO SINGULAR. MOSTROU-SE A EVOLUÇÃO \\
DA INTEGRALIDADE DA ASSISTÊNCIA ATRAVÉS DO \\
APOIO MATRICIAL E DAS FORMAS DE QUALIFICAR A \\
EQUIPE DA UBS QUE, POR MEIO DE UMA FERRAMENTA \\
A MAIS COM ATIVIDADES EXTRAMUROS, PODE \\
PROPORCIONAR GRANDES GANHOS NA ASSISTÊNCIA A \\
POPULAÇÃO.
\end{tabular}

Fonte - Dados coletados pelas pesquisadoras, Fortaleza, Ceará, Brasil, 2021.

Os estudos selecionados evidenciaram perspectivas diversas do cotidiano dos ACS em variados planos, compondo uma bagagem de vertentes importantes para possibilitar interpretações amplas e, consequentemente, resolubilidade maior das questões aqui levantadas. A partir da análise dos dados, emergiram as categorias temáticas: "A importância do agente comunitário de saúde no matriciamento", "O cuidado em saúde mental dos agentes comunitários de saúde" e "O apoio matricial como ferramenta de capacitação".

\section{DISCUSSÃO}

\section{A IMPORTÂNCIA DO AGENTE COMUNITÁRIO DE SAÚDE NO MATRICIAMENTO}

É consenso entre os autores o papel estratégico do ACS no apoio matricial. Por serem os maiores conhecedores das problemáticas sociais que atingem a vida das pessoas da comunidade, podem identificar fatores potenciais para $\mathrm{o}$ sofrimento psíquico, e assim propiciar escuta, além de orientações sobre assistência na rede ${ }^{8-13}$.

$\mathrm{O}$ apoio matricial configura-se como ferramenta premente na manutenção de cuidados às pessoas que demandam cuidados em saúde mental no território, pois contribui de maneira singular com a evolução da assistência em saúde integral e humanizada, por meio do amparo aos profissionais 
da APS. A partir do comportamento dinâmico e de muitas perspectivas no olhar do ACS e da equipe de matriciadores, é possível reinventar as estratégias para alcançar melhores resultados de acordo com as demandas de cada usuário e equipe de saúde ${ }^{11}$.

Em um ambiente complexo e rodeado por circunstâncias do contexto de vida dos pacientes, o apoio matricial se mostra potente na formulação de um cuidado singular e efetivo por meio dos conhecimentos compartilhados entre os profissionais de referência e os profissionais da UBS, definindo fluxos e alicerçando a assistência desses pacientes dentro do seu território através da corresponsabilização entre essa ${ }^{13}$. Desse modo, inibindo a saturação da atenção secundária, garantindo o atendimento às demandas de diversos graus de complexidade em seus respectivos serviços, dentro de suas capacidades de acolhimento e manutenção do cuidado ${ }^{8}$.

Embora já exista uma grande consciência a respeito da importância da saúde mental no ambiente da UBS, ainda é de difícil estruturação o matriciamento nessas unidades por diversos fatores. Silva, Santos e Souza ${ }^{12}$ citam dentre esses obstáculos a falta de recurso estrutural, mudança frequente dos integrantes da equipe e dificuldades de meios de interlocução efetivos e acessíveis aos profissionais, comprometendo uma importante tecnologia leve de cuidado, a comunicação ativa.

\section{O CUIDADO EM SAÚDE MENTAL DOS AGENTES COMUNITÁRIOS DE SAÚDE}

São amplas e diversas as demandas de saúde mental na atenção primária, o que produz angústias e tensões nos ACS, bem como em outros integrantes da equipe de Saúde da Família. Tais sensações culminam em alguns procedimentos mecanizados verticalizados ${ }^{12}$.

Amaral, Torrente, Torrente e Moreira ${ }^{8}$ descrevem sobre o medo em relação à loucura e a ausência de reconhecimento dessas demandas como de reponsabilidade da equipe de atenção primária. O manejo de um caso de sofrimento psíquico pode gerar ansiedades no ACS. Há o receio de comportamentos imprevisíveis, o despreparo e a falta de recursos para ajudar, ao mesmo tempo que se posicionar como um cuidador em saúde mental coloca em questão suas próprias fragilidades ${ }^{12}$.

Desse modo, o matriciamento possibilita uma mudança na percepção de tais fatos, a partir da maior compreensão sobre o que são os transtornos mentais, descoberta de possibilidades de intervenção e o contato dos profissionais com tais demandas, facilitando a desmistificação da loucura $^{8}$.

A partir do matriciamento, os ACS passam a dar novos sentidos às suas práticas, reconhecendo-se com sujeitos de seu próprio saber e por esse motivo mais qualificados para realizarem o cuidado em saúde mental. O espaço onde o ACS vive é o mesmo onde atua, sendo desse modo, o cuidado direcionado às pessoas com quem ele convive na comunidade. Tal fato propicia uma um sentimento de identificação com suas histórias, angústias e reivindicações ${ }^{12}$.

Silva, Santos e Souza ${ }^{12}$ corroboram os achados de Campos et al. ${ }^{13}$, ao pontuarem que os ACS já realizam o cuidado em saúde mental de forma intuitiva nas visitas domiciliares, permeados pela sensibilidade e conhecimento de senso comum, este sendo visto como alicerce para a reconstrução do saber. O encontro e o diálogo com a comunidade aproximam o ACS da realidade local. E as suas experiências possibilitam a produção do cuidado. Assim, o profissional também aperfeiçoa sua prática. A partir da convivência com o outro, vive a produção pedagógica ao mesmo tempo em que produz cuidado.

A variedade de percepções do ACS sobre o atendimento e abordagem ao paciente em sofrimento psíquico mostra a gama de habilidades necessárias para a realização desse manejo. Santos e Nunes ${ }^{11}$ versam que em alguns ACS ainda prevalece um discurso pautado no modelo biomédico, centrado na patologia, sintomas, 
mantendo ainda uma visão estigmatizada da assistência em saúde mental, desencadeando prejuízos no desempenho de estratégias de cuidado e corresponsabilização.

Apesar dessa dificuldade, o profissional ACS se mostra consciente de seu papel nessa prática de cuidado. Por meio do conhecimento amplo sobre o contexto de vida de cada indivíduo do território, a proximidade com o ambiente familiar e social e a história pregressa de saúde do indivíduo e coletividade, o ACS tem informações importantes para a estruturação do perfil do paciente, suas principais necessidades. Com isso, auxilia a equipe de APS no planejamento de terapêutica singular efetiva. Com a utilização de alguns instrumentos do cotidiano desse profissional, como visita domiciliar e busca ativa, é possível mapear os casos até o momento desconhecidos e organizar a dinâmica de assistência à saúde mental de acordo com o perfil de cada sujeito ${ }^{9,10}$.

Nesse contexto, constituindo a equipe de Saúde da Família na APS, o agente comunitário de saúde acaba por se comprometer com várias de suas habilidades e procedimentos de rotina no amparo a indivíduos em processo de sofrimento psíquico. Dentro dessa condição, torna-se primordial o amparo por meio da capacitação desse profissional, que atua de maneira árdua no território e consegue observar de perto aspectos bem distantes do olhar de outros profissionais da estratégia ${ }^{8,11}$.

\section{O APOIO MATRICIAL COMO FERRAMENTA DE CAPACITAÇÃO}

Como estratégia de educação permanente em saúde, o matriciamento realizado por uma equipe especializada pode conduzir o ACS a muitos ganhos no âmbito do conhecimento, desenvolvendo uma escuta qualificada e desencadeando uma cadeia de procedimentos positivos tanto para a população quanto para o sistema de saúde ${ }^{9}$. A partir do treinamento e das experiências subsequentes desses com o público, o que possibilita um sentido vivencial aos conceitos e a incorporação na atuação profissional, o ACS consegue construir um alicerce para as abordagens e conduções à população que necessita de cuidados em saúde mental no território ${ }^{12}$.

A experiência do ACS no apoio matricial possibilita a compreensão sobre a integralidade do cuidado, o conceito ampliado de saúde. As discussões com os matriciadores apontam a necessidade de conhecer as ações intersetoriais, o que implica na diversificação de manejos do cuidado em saúde mental, rompendo a lógica mecanizada de assistência, bem como o maior envolvimento do indivíduo e família no tratamento 9,13

A visibilidade e presença desses profissionais nos serviços de referência são estratégias de melhorias para o acesso aos serviços de saúde. Quando o ACS conhece os outros serviços de saúde mental, além dos hospitais e outros equipamentos ambulatoriais e de internação, possibilita educar a comunidade, pois a forma como os indivíduos e a família compreendem a atuação dos serviços especializados, neles incluindo o CAPS, é determinante para a busca e a adesão ao tratamento, desconstruindo a visão estigmatizada dos serviços de saúde mental ${ }^{8}$.

Ademais, a parceria estruturada e frequente com os serviços especializados de referência, como CAPS e NASF, possibilita a discussão compartilhada de casos, intervenções conjuntas no território, atendimento conjunto, corresponsabilização e pactuações de projeto terapêutico singular de acordo com as necessidades do momento. Tais estratégias aumentam a capacidade resolutiva da APS, amplia a clínica e capilariza os saberes técnicos ${ }^{13}$.

Além disso, Moura e Silva ${ }^{10}$ destacam que, com a qualificação de profissionais atuantes na assistência a pacientes em sofrimento psíquico, assegura-se o direito e a proteção desse público de ser atendido em comunidade e ser reinserido na sociedade.

Consoante com o cuidado coordenado em rede, o apoio matricial desperta nos ACS a 
valorização da solidariedade e do exercício da democracia. A educação direcionada a esses trabalhadores, além de promover capacitação para atuação qualificada em saúde mental, contribui para o empoderamento da comunidade. Assim, o ACS estimula a crítica e a autonomia dos usuários, direcionando-os a encontrar na própria comunidade formas para lidar com seus problemas, transformando ativamente $\mathrm{o}$ processo de construção da saúde ${ }^{12}$.

Logo, diante da dinâmica de trabalho do profissional ACS, que atua de maneira árdua no território, sendo a ponte forte e acessível através da sua interlocução simples e adequada com a população, a capacitação profissional aproxima uma assistência integral e integrada para a população e entre os serviços, que muito tem a ganhar com o diálogo entre os profissionais de diferentes serviços e níveis de complexidade, que juntos podem construir novas dimensões de cuidar, nessa vertente tão ampla e complexa que é a saúde mental ${ }^{9,11}$.

\section{CONSIDERAÇÕES FINAIS}

Desse modo, ressalta-se a importância do enlace entre o matriciamento, atenção primária à saúde e o agente comunitário de saúde. Uma tríade que se explorada de maneira bem planejada e com valorização pode produzir melhorias e impactos positivos na assistência em saúde mental da população e no cotidiano de trabalho de profissionais de saúde dentro do território. Ao realizar a busca de estudos, teve-se como maior desafio a limitação do número de produções mais atuais e que estivessem com seu texto disponível na íntegra de forma gratuita e on-line, reduzindo o acesso a uma maior quantidade de informações para a construção dessa produção. Além de ser uma temática ainda pouco explorada na construção de estudos, conduzindo a sua condição de escassez apesar da imensa importância da estruturação de produções sobre o tema para subsidiar os interesses assistenciais.
Assim, com o estabelecimento da assistência articulada e organizada, propicia-se a integralidade do cuidado com corresponsabilização.

Por meio dessa integralidade, pode-se vislumbrar uma assistência mais humanizada, assertiva e que produza de fato bons resultados dentro daquilo que se propõe no plano terapêutico singular de cada usuário do serviço, que depende diretamente da qualidade de assistência que o serviço oferece, independente do seu nível de atenção dentro do sistema de saúde.

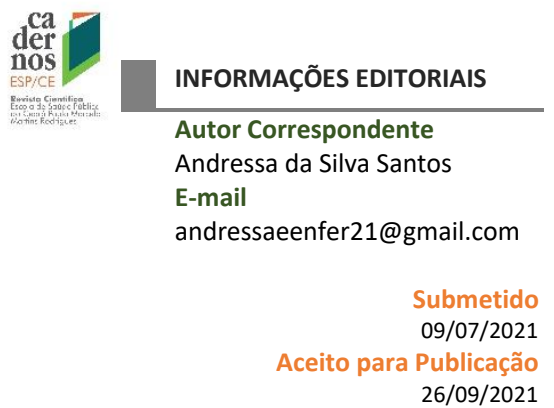




\section{REFERÊNCIAS}

1.Firmino DG, Lôbo APA. Atuação dos enfermeiros em Saúde Mental na Estratégia Saúde da Família no Município de IcapuíCE. Cadernos ESP. 2019;13(1):9-18. ISSN: 1808-7329/1809-0893.

2.Santos RC, Bosi MLM. Saúde Mental na Atenção Básica: perspectivas de profissionais da Estratégia Saúde da Família no Nordeste do Brasil. Ciência Saúde Col [online]. 2021; 26(5): 1739-48. doi 10.1590/1413-81232021265.04902021.

3.Secretaria de Atenção à Saúde (BR). Departamento de Atenção Básica. Núcleo de Apoio à Saúde da Família: Ferramentas para a gestão e para o trabalho cotidiano. Ministério da Saúde. Caderno de Atenção Básica. Brasília. 2014; 1(39). Disponível em: https://bvsms.saude.gov.br/bvs/publicacoes/nucleo_apoio_saude_familia_cab39.pdf.

4. Ministério da Saúde (BR). Centro de Estudo e Pesquisa em Saúde Coletiva. Guia prático de matriciamento em saúde mental / Dulce Helena Chiaverini (Organizadora) et al. Brasília; 2011. Disponível em: https://bvsms.saude.gov.br/bvs/publicacoes/guia_pratico_matriciamento_saudemental.pdf.

5.Onocko-Campos RT, Campos GWS, Ferrer AL, Corrêa CRS, Madureira PR, Gama CAP, Dantas DV, Nascimento R. Avaliação de estratégias inovadoras na organização da Atenção Primária à Saúde. Rev Saúde Pública [online]. 2012;46(1): 43-50. doi 10.1590/S0034-89102011005000083.

6.Cabral TMN, Albuquerque PC. Saúde mental sob a ótica de Agentes Comunitários de Saúde: a percepção de quem cuida. Saúde em Debate [online]. 2015;39(104): 159-71. doi 10.1590/0103-110420151040415.

7.Souza MT, Silva MD, Carvalho R. Revisão integrativa: O que é? Como fazer isso? Einstein [online]. 2010;8(1):102-106. doi 10.1590/S1679-45082010RW1134.

8.Amaral CDM, Torrenté MON, Torrenté M, Moreira CP. Apoio matricial em Saúde Mental na atenção básica: efeitos na compreensão e manejo por parte de agentes comunitários de saúde. Interface - Comunicação, Saúde, Educação [online]. 2018;22(66):801-812. doi 10.1590/1807-57622017.0473.

9.Silva MAC, Aguiar MGG, Moreira TDS. Entre os nós da de saúde mental: As Práticas de Agentes Comunitários de Saúde. Rev Baiana Saúde Pub. 2016;40(3):713-28. doi 10.22278/2318-2660.2016.

10.Moura RFS, Silva CRC. Saúde Mental na Atenção Básica: Sentidos Atribuídos pelos Agentes Comunitários de Saúde. Psicologia: Ciência e Profissão [online]. 2015;35(1):199-210. doi 10.1590/1982-3703001832013.

11.Santos GA, Nunes MO. O cuidado em saúde mental pelos agentes comunitários de saúde: o que aprendem em seu cotidiano de trabalho?. Physis: Rev Saúde Col [online]. 2014;24(01): 105-25. doi 10.1590/S0103-73312014000100007.

12.Silva CB, Santos JE, Souza RC. Estratégia de apoio em saúde mental aos agentes comunitários de saúde de Salvador-BA. Saúde Soc [online]. 2012;21(1):153-60. doi 10.1590/S0104-12902012000100015.

13.Campos RO, Gama CA, Ferrer Al, Santos DVD, Stefanello S, Trapé TL, Port K. Saúde mental na atenção primária à saúde: estudo avaliativo em uma grande cidade brasileira. Ciência Saúde Col [online]. 2011;16(12):4643-52. doi 10.1590/S141381232011001300013. 